\title{
“Carpa de protección solar”. La dermofarmacia como herramienta para la concienciación y prevención del cáncer de piel.
}

Uriel Gallego, $M^{\mathrm{a}}$; Sáez-Benito Suescun, A $^{\mathrm{b}}$; Gómez Rincón, $C^{\mathrm{c}}$.

${ }^{a}$ Universidad San Jorge, muriel@usj.es ${ }^{\mathrm{b}}$ Universidad San Jorge, amsaezbenito@usj.es ${ }^{\mathrm{c}}$ Universidad San Jorge, cgomez@usj.es.

\begin{abstract}
During academic year 2017/2018 the project: "Sun damage protection tent" take place as a Service-Learning Methodology. In this experience, diverse agents are involved, creating an interdisciplinary space in which pharmacy students are going to participate.
\end{abstract}

This project is presented in order to motivate the students to create a didactic material, a leaflet, and a website with the awareness campaign ideas to present in the annual "Sun damage protection tent" that promotes the "Colegio Oficial de farmacéuticos de Zaragoza"(C.O.F.)and "Asociación Española Contra el Cáncer”(A.E.C.C.)

Pharmacy students are going to be the creative force of the campaign. Also, they are going to be part of the participating volunteers of the "Sun damage protection tent" and to complete the activities of the project, it will be close with the location of a "sun tent" on Universidad San Jorge's campus. There, the students can share the knowledge acquired during the experience, their communication skills and try to educate their colleagues and other member of the academic staff in sun protection.

The participation of the students will consist in the design, training, diffusion, implementation and assessment of the project. Varied innovative methodologies are used in this experience: Service-Learning and Information and communications technology.

\section{Resumen}

Durante el curso académico 2017/2018 se lleva a cabo el servicio de Aprendizaje-Servicio "Carpa de protección solar". La dermofarmacia como herramienta para la concienciación y prevención del cáncer de piel. En esta experiencia intervienen diferentes agentescreando un espacio interdisciplinar en el que van a participar los estudiantes de farmacia.

El presente proyecto motiva a los alumnos a crear un material didáctico, un folleto y una página web que contengan las principales ideas de la campaña anual "Carpa solar" que el Colegio Oficial de Farmacéuticos de 
“Carpa de protección solar”. La dermofarmacia como herramienta para la concienciación y prevención del cáncer de piel.

\section{Zaragoza(C.O.F.) organiza junto ala Asociación Española contra el Cánces} (A.E.C.C.)

Los alumnos van a formar parte de la de la campaña desde su creación hasta la participación como voluntarios en las carpas solares. Para completar el proyecto, se finalizará con la instalación de una carpa solar en el campus universitario. Los estudiantes podrán compartir su aprendizaje adquirido sobre protección solar, tanto con sus compañeros de otros grados como con el personal académico.

La participación de los alumnos consistirá en el diseño, práctica, difusión y evaluación del proyecto. Se aplican metodologías innovadoras como el Aprendizaje-Servicio(APS) y las tecnologías de la Información y la Comunicación (TICs)

Palabras clave: Aprendizaje en Dermofarmacia, Aprendizaje Servicio, TICs, Carpa Solar

\section{Introducción}

Uno de los temas fundamentales en la asignatura de dermofarmacia, es la prevención de los daños solares mediante el uso de preparados dermocosméticos y consejos farmacéuticos. Para el curso 2017/2018, se plantea el proyecto: "Carpa de protección solar. La dermofarmacia como herramienta para la concienciación y prevención del cáncer de piel”, como una metodología de aprendizaje servicio, en el que van a participar diversos agentes, generando un espacio interdisciplinar del que van a ser partícipes los alumnos de dermofarmacia. Además de tratar el tema desde un punto de vista teórico y completarlo con prácticas de laboratorio y casos prácticos de resolución en clase, se plantea el proyecto a través de la motivación al alumno a crear un material didáctico tipo blended learning, junto a un tríptico con un resumen informativo y un anuncio/cartel. Todo esto, será presentado a la campaña de protección solar que el Colegio Oficial de Farmacéuticos de Zaragoza organiza todos los años junto a la Asociación Española Contra el Cáncer. Con esta colaboración, los alumnos de farmacia de la USJ, van a convertirse en el motor creativo de la campaña del 2018, además de pasar a formar parte de los voluntarios que participen en las carpas solares organizadas en piscinas y puntos urbanos. Para completar las actividades, el proyecto se cerrará con la ubicación de una carpa solar en el campus de la Universidad San Jorge, donde los alumnos de dermofarmacia, compartirán los conocimientos adquiridos, comunicándolos e informando a los estudiantes de otros grados y personal universitario y realizando analíticas de pigmentación de piel y protección requerida mediante el manejo del equipo "dermoanalizador de piel”. La participación de los alumnos consistirá en el diseño del proyecto, la formación, difusión, puesta en marcha y evaluación del mismo. Esto va a ofrecer una visión global de cómo el aprendizaje puede situarse y aplicarse fuera del aula y ofreciendo un servicio a la población.

(cc) EY-NC-ND 2018, Universitat Politècnica de València

Congreso IN-RED (2018) 
La concienciación para la protección solar es un tema en el que el farmacéutico juega un rol fundamental. Como profesionales accesibles a la población, el estudiante de farmacia debe familiarizarse con la participación en esta y otras campañas de prevención, y que mejor forma de hacerlo que la propia intervecnción en un proyecto que combina las metodologías de docencia: Aprendizaje Servicio (APS) y la inclusión de las Tecnologías de la Información y Comunicaciones (TIC).

Las propuestas de aprendizaje servicio (APS), se situan en el marco de un modelo formativo de universidad que procura combinar aprendizaje académico y formación para una ciudadanía activa en tiempo real.

Las propuestas de APS no deben confundirse con la prestación de servicios voluntarios. Deben suponer aprendizaje académico en los estudiantes $\rightarrow$ Aprendizaje y contribuir a la mejora de la calidad de vida y del nivel de inclusión social en la población $\rightarrow$ Servicio.(Martínez, 2008)

En este proyecto se definen ambas partes:

-Aprendizaje: dermoprotección, daños solares y sus posteriores patologías, productos para la protección solar e indicaciones para su aplicación.

-Servicio: Transmisión a la sociedad que rodea a los alumnos participantes en el proyecto de la importancia de conocer los mecanismos para la prevención de los daños ocasionados de la exposición al sol. Consejos y detección de peligros de la radiación solar.

Las Tecnologías de la Información y Comunicaciones TIC han devenido incorporándose naturalmente en ladocencia universitaria obedeciendo a distintas motivaciones. El énfasis está en usarlas de apoyo en elproceso docente tradicional. Por sí mismas las TIC e Internet no educan ni reemplazan al profesor, son un recurso poderoso cuya incorporación debe considerar nuevos roles del profesor, nuevos contenidos, programas y formas de evaluar.(Vera, 2003)( Muñoz-Repiso, 2014)

\section{Objetivos}

- Reforzar desde el aprendizaje práctico y la transmisión de conocimientos, el estudio de los alumnos sobre los riesgos del sol y los beneficios de la prevención como herramienta dermofarmacéutica.

- Aplicar el conocimiento adquirido a un ámbito real. Motivar al alumno para que sea consciente de la importancia de su aprendizaje para su ejercicio profesional, y como servicio a la población.

- Diseñar un material didáctico elaborado por el propio alumno y que va a tener varios destinatarios: el propio alumno, sus compañeros y el resto de participantes en la campaña: farmacéuticos y voluntarios, pero sobre todo un destinatario final: la población.

- Practicar los recursos comunicativos empleados por el alumno, para la difusión del conocimiento a la población, tan importante en su futuro profesional como farmacéutico.

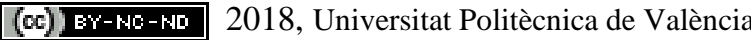


“Carpa de protección solar”. La dermofarmacia como herramienta para la concienciación y prevención del cáncer de piel.

- Involucrar al alumno como un agente fundamental en una actividad profesional y de servicio a la comunidad.

\section{Desarrollo de la innovación}

En el presente proyecto, se trata de aplicar metodologías impulsadas por la Universidad San Jorge con el fin de ofrecer un modelo educativo que proporcione "una experiencia única y transformadora de la persona” y que alcance impactos positivos en el aprendizaje de los alumnos.

Se emplea la metodología de aprendizaje servicio (APS). Se trata de que el alumno aprenda mientras participa en las diferentes fases del proyecto: en el diseño y creación del material didáctico, y en su puesta en práctica en un contexto real como es una carpa de concienciación al público, un contexto que va mucho más allá del aula.

Debido a las características del proyecto, se pretende concienciar al alumno con su contexto social inmediato; entendiendo que con su transmisión de conocimiento, está llevando a cabo un servicio a la ciudadanía, reforzando con ello su vocación sanitaria profesional.

Se trata de integrar las tecnologías de la información y la comunicación (TIC) durante todo el proyecto: creación de material didáctico, diseño de una página web, creación de vídeos, diseño de material gráfico, tipo cartel/portada para la difusión (comunicación) por diferentes medios: webs, aplicaciones móviles, redes sociales, prensa...

Se suma al punto anterior, la metodología de enseñanza de uso y aplicación de un equipo como es el "analizador de piel", con un fin concreto y aplicado, que pretende abrir la mente a las numerosas aplicaciones que se pueden conseguir con las herramientas que en su futuro profesional, el farmacéutico puede llegar a tener a su alcance, y cómo podrá utilizarlas, en beneficio del paciente.

En este proyecto, se trabaja con una asociación de pacientes, con un colegio profesional, y de la mano de farmacéuticos colegiados, futuros colegas de los alumnos. Además se trabaja para poder ofrecer una información a pacientes, a otros alumnos de su mismo y otros grados. Esto hace que dicha colaboración interdisciplinar y con otros agentes sociales, repercuta de manera positiva en el aprendizaje del estudiante de dermofarmacia, que entienda los beneficios directos de la adquisición de conocimientos. Todo ello, en una ubicación fuera del aula.

\subsection{Contextualización}

A continuación se describen los destinatarios, beneficiarios de este proyecto.

Desde el punto de vista del aprendizaje, con toda su implicación en la parte docente: los alumnos de la asignatura Dermofarmacia y cosmética; impartida en $5^{\circ}$ curso y los de Inmunología impartida en $3^{\circ}$ curso del Grado de Farmacia de la Facultad de Ciencias de la Salud. Universidad San Jorge.

(cc) EY-NC-ND 2018, Universitat Politècnica de València

Congreso IN-RED (2018) 
Los destinatarios del servicio: la asociación de pacientes de cáncer (AECC), la población en general participante en las carpas solares y particularmente los participantes del campus, compañeros y trabajadores de la Universidad.

Cabe resaltar la colaboración con el Colegio Oficial de Farmacéuticos de Zaragoza y el contacto de los alumnos con los farmacéuticos colegiados, futuros compañeros de profesión.

\subsection{Cronograma}

Todas estas actividades se llevarán a cabo según el cronograma descrito en la tabla 1:

Tabla 1. Cronograma de actividades del proyecto

\begin{tabular}{|c|c|c|c|c|c|c|c|c|c|c|c|}
\hline ACTIVIDAD & $\begin{array}{l}09 / \\
17\end{array}$ & $\begin{array}{c}10 / \\
17\end{array}$ & $\begin{array}{l}11 / \\
17\end{array}$ & $\begin{array}{c}12 / \\
17\end{array}$ & $\begin{array}{r}01 / \\
18\end{array}$ & $\begin{array}{c}02 / \\
18\end{array}$ & $\begin{array}{c}03 / \\
18\end{array}$ & $\begin{array}{c}04 / \\
18\end{array}$ & $\begin{array}{r}05 / \\
18\end{array}$ & $\begin{array}{c}06 / \\
18\end{array}$ & $\begin{array}{r}07 / \\
18\end{array}$ \\
\hline $\begin{array}{c}\text { Presentación del proyecto a } \\
\text { alumnos }\end{array}$ & $\mathrm{X}$ & & & & & & & & & & \\
\hline $\begin{array}{c}\text { Trabajo grupal en los vídeos } \\
\text { (Inmunología 3ㅇ) }\end{array}$ & & $X$ & $x$ & & & & & & & & \\
\hline $\begin{array}{c}\text { Evaluación de los vídeos } \\
\text { (rúbrica) por docentes y } \\
\text { alumnos de 5ㅇ }\end{array}$ & & & $x$ & $X$ & & & & & & & \\
\hline $\begin{array}{l}\text { Trabajo grupal de creación del } \\
\text { material didáctico prevención y } \\
\text { riesgo solar (Dermofarmacia 5ㅇ) }\end{array}$ & & $X$ & $X$ & $\mathrm{X}$ & & & & & & & \\
\hline $\begin{array}{l}\text { Evaluación del material } \\
\text { didáctico carpa solar por } \\
\text { docentes y COF Zaragoza }\end{array}$ & & & & $\mathrm{X}$ & $X$ & & & & & & \\
\hline $\begin{array}{l}\text { Diseño y creación continua de } \\
\text { la página web del proyecto }\end{array}$ & & $x$ & $x$ & $x$ & $x$ & $x$ & $x$ & $x$ & $x$ & $\mathrm{X}$ & \\
\hline $\begin{array}{c}\text { Evento: carpa solar en Zaragoza } \\
\text { y carpa solar USJ }\end{array}$ & & & & & & & & & $x$ & $x$ & \\
\hline $\begin{array}{c}\text { Evaluación del aprendizaje y } \\
\text { servicio llevado a cabo durante } \\
\text { las carpas }\end{array}$ & & & & & & & & & $x$ & $x$ & $X$ \\
\hline $\begin{array}{l}\text { Evaluación de resultados y } \\
\text { difusión del proyecto }\end{array}$ & & & & & & & & & & $\mathrm{X}$ & $X$ \\
\hline
\end{tabular}


“Carpa de protección solar”. La dermofarmacia como herramienta para la concienciación y prevención del cáncer de piel.

\subsection{Evaluación}

Para el seguimiento y calificación del proyecto, se emplean diferentes tipos de evaluación, llevadas a cabo por diferentes agentes participantes. Con ello se pretende incluir la evaluación como parte del aprendizaje; por ejemplo con la evaluación dinámica, autoevaluación, o con la inclusión de los alumnos en la evaluación de los trabajos grupales.

Autoevaluación: los alumnos al finalizar el proyecto realizan una autoevaluación que permite conocer su aprendizaje y la repercusión que ha tenido el proyecto.

La forma en que los alumnos participan en la evaluación de sus compañeros es que los alumnos de $5^{\circ}$ curso de dermofarmacia, quienes ya han superado la asignatura de inmunología, realizan una evaluación de la tarea grupal de creación de vídeos llevada a cabo por sus compañeros de tercer curso. Esta evaluación se realiza mediante una rúbrica proporcionada por los docentes del proyecto, además pueden aportar comentarios a cada apartado evaluado. Los alumnos de quinto acceden a los vídeos mediante la web del proyecto. (Casanova, 2009)

Los participantes también pueden llevar a cabo una evaluación del proyecto aunque esta es opcional.

\section{Resultados}

Tal como se muestra en el cronograma, el proyecto se encuentra todavía en ejecución, por lo que algunos resultados no se encuentran finalizados.

Documentación del proyecto y productos generados:

-Website del proyecto:

Toda esta información creada por los alumnos, se recoge en web

https://sites.google.com/usj.es/carpasolar/

-Videos informativos/educativos:

Estos vídeos fueron creados por los alumnus de tercer curso y evaluados por sus compañeros de quinto curso,además de por el profesorado;

https://sites.google.com/usj.es/carpasolar/página-principal/vídeos

- Panfleto para la difusión de la campaña, ver Figura 1.-: 

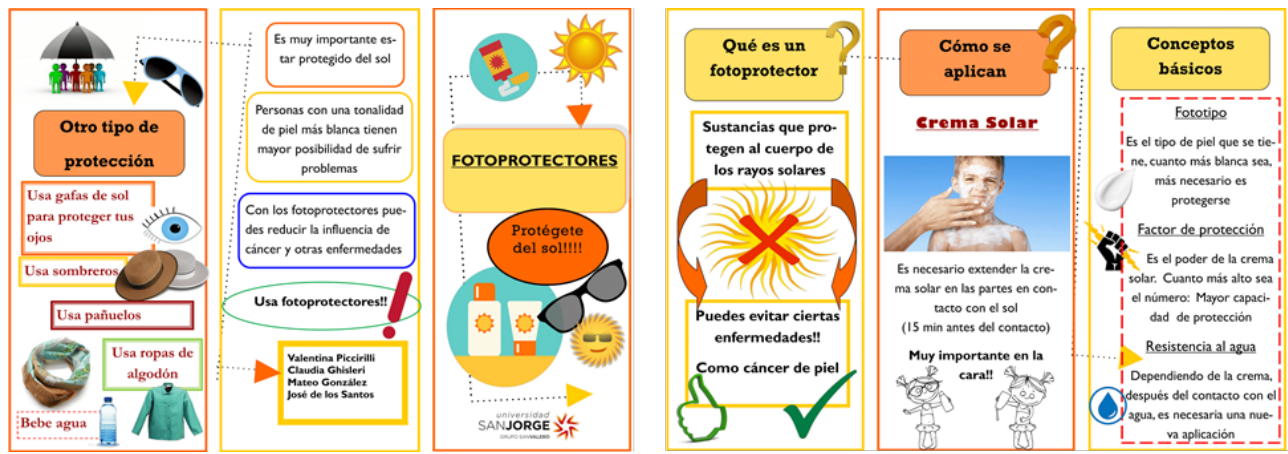

Fig. 1 Imagen de un folleto informativo creado por alumnos para la carpa solar

Resultados de evaluación del proyecto y rúbricas de evaluación.

La participación y motivación de los alumnos en la creación tanto del material, vídeos, así como de la página web es alta.

Actualmente contamos con los resultados de la evaluación entre alumnos: la que realizada por los alumnos de dermofarmacia de $5^{\circ}$ a los 13 vídeos elaborados por los de inmunología de $3^{\circ}$ recogida en la Figura 2.-

Se recogen las notas medias de dichas evaluaciones, obtenidas mediante una rúbrica creada por los docente y que los alumnos de dermofarmacia debán colgar completa con las notas y sus comentarios en la Plataforma Docente Universitaria.

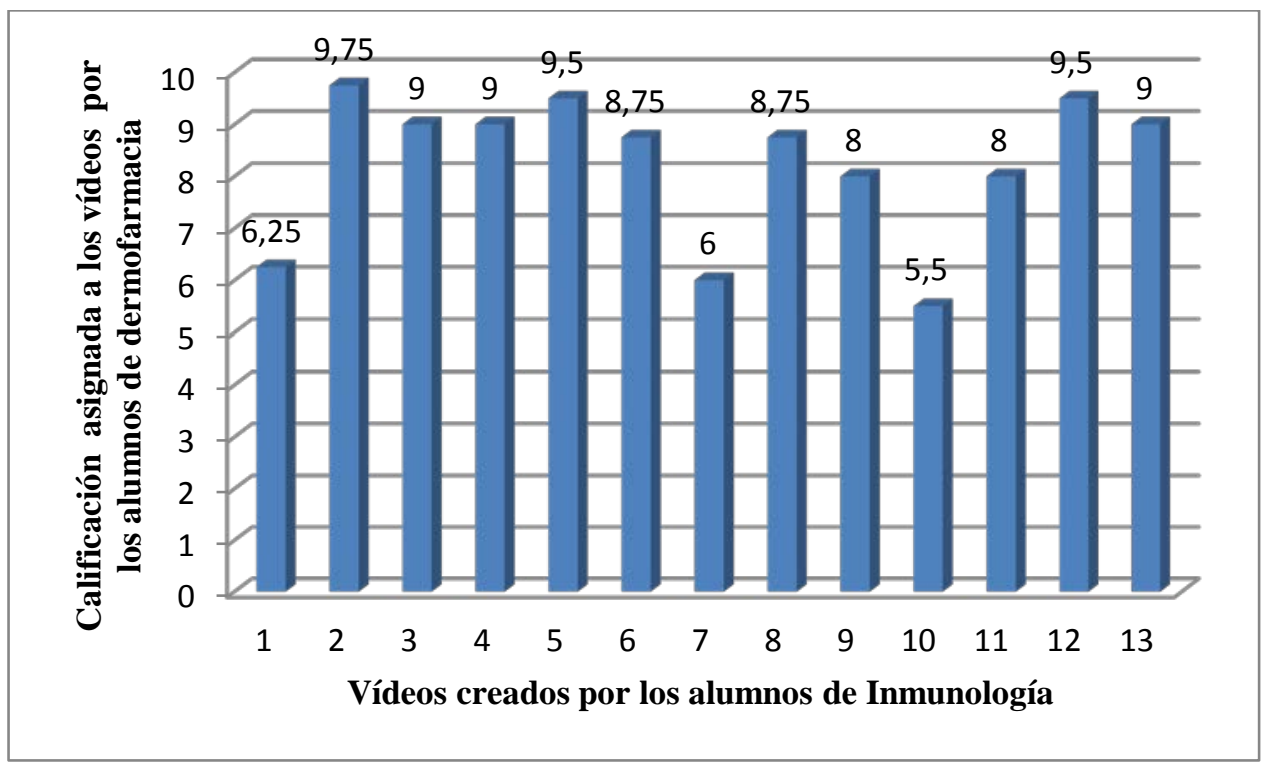

Fig.2 Gráfica de la media de los resultados de la evaluación realizada por los alumnos de $5^{\circ}$ sobre los 13 vídeos creados por los alumnos de Inmunología de $3^{\circ}$.

(c)) EY-NC-ND 2018, Universitat Politècnica de València

Congreso In-Red (2018) 
“Carpa de protección solar”. La dermofarmacia como herramienta para la concienciación y prevención del cáncer de piel.

La evaluación llevada a cabo por los docentes también se realizó mediante una rúbrica, y en el caso de la asignatura dermofarmacia, los alumnos obtuvieron una nota media de 8,8/10 frente al 6,5/10 de nota media obtenida en el total de la asignatura.

\section{Material para la difusión del proyecto:}

- Documentación en formato artículo.

- Póster o comunicación oral con los resultados del proyecto y divulgación de los mismos.

\section{Conclusiones}

Los alumnos han estado muy motivados con el proyecto y las calificaciones de las que se disponen están por encima de la media obtenidas en las asignaturas, destacamos que la nota media del proyecto en la asignatura de dermofarmacia está 2,3/10 puntos por encima de la media total de dicha materia.

Con este proyecto se consigue que los futuros farmacéuticos alcancen el aprendizaje relacionado con la protección solar y la prevención de los riesgos solares mediante su ejercicio profesional en actividades en las que practican la comunicación con el paciente, la generación de información, el análisis de la piel y el consejo dermofarmacéutico. Todo esto a su vez, genera un servicio a la sociedad y una ayuda a la asociación contra el cáncer que da el sentido a todo el proyecto. Se consigue por tanto, el ansiado tándem aprendizajeservicio que logra motivar y apasionar a todos los agentes implicados.

\section{Referencias}

MARTÍNEZ M. (2008) "Aprendizaje servicio y construcción de ciudadanía activa en la universidad: la dimensión social y cívica de los aprendizajes académicos”. Aprendizaje servicio y responsabilidad social de las universidades, 11-26.

GINNS, \& R. A. ELLIS P. (2009) "Evaluating the quality of e-learning at the degree level in the student experience of blended learning”. British Journal of Educational Technology, 40(4), 652-663.

BIGGS J., BIGGS J. B.(2006) Calidad del aprendizaje universitario. Narcea ediciones.

VERA A. (2003) "Las tecnologías de información y comunicaciones (TIC) en la docencia universitaria”. Theoría: Ciencia, Arte y Humanidades, 12, 109-118.

MUÑOZ-REPISO A. G. V. (2014) Procesos de innovación didáctica basados en el uso de las nuevas tecnologías: En Experiencias de Innovación Docente Universitaria. Ediciones Universidad de Salamanca.

HERNÁNDEZ F., GARCÍA M., MAQUILÓN J. J. (2014) "Estudio empírico de los enfoques de aprendizaje de los estudiantes universitarios en función del perfil de su

(cc) EY-NC-ND 2018, Universitat Politècnica de València

Congreso IN-RED (2018) 
titulación (profundo VS superficial) = Empirical research on university students' learning approaches depending on the degree undertaken”. REOP-Revista Española de Orientación y Psicopedagogía, 12(22), 303-318.

CASANOVA J. M., BARADAD M., SORIA X., \& MARTÍ R. M. (2009) “www. dermatoweb. net. Una web docente para el aprendizaje de la Dermatología en el pregrado”. Actas Dermo-Sifiliográficas, 100(10), 866-874.

PÉREZ V., MANUEL J., O.DELGADO QUINTANA M., GARCÍA LÓPEZ I., GARCÍA MÁRQUEZ J. A., SABORIDO CEBALLOS A., \& FERNÁNDEZ ARÉVALO M. M. (2010) Facultad de Farmacia de la Universidad de Sevilla: 4 Años de plan piloto para la adaptación al EEES. Actividades docentes realizadas para una mejora en la calidad de enseñanza y su repercusión en el alumnado. 